\title{
IMPACT OF VARIOUS SOURCE OF COVARIANCE INFORMATION ON INTEGRAL PARAMETERS UNCERTAINTY DURING DEPLETION CALCULATIONS WITH CASMO-5
}

\author{
Mathieu Hursin ${ }^{1,2 *}$, Dimitri Rochman ${ }^{1}$, Alexander Vasiliev ${ }^{1}$, \\ Hakim Ferroukhi ${ }^{1}$ and Andreas Pautz ${ }^{1,2}$ \\ ${ }^{1}$ Paul Scherrer Institut, Nukleare Energie und Sicherheit \\ PSI Villigen 5232, Switzerland
}

${ }^{2}$ Ecole Polytechnique Fédérale de Lausanne, Laboratory for Reactor Physics and Systems behaviour Lausanne 1015, Switzerland

mathieu.hursin@psi.ch,dimitri-alexandre.rochman@psi.ch, alexander.vasiliev@psi.ch, hakim.ferroukhi@psi.ch, andreas.pautz@psi.ch

\begin{abstract}
This paper describes the effect of input uncertainties on a set of integral parameters $(\mathrm{k}-$ inf, nuclide compositions) associated with the validation of CASMO-5 against PIE data. The nuclear data under consideration are the cross-sections, fission spectrum and neutron multiplicities and fission yields. Various sources of covariance information are considered, novel ones (ENDFB-VIII.0, JEFF-3.3) as well as more widely distributed ones (JENDL-4.0, ENDF/B-VII.1, Scale 6.1 and Scale 6.2). All possible nuclide reaction pairs (cross sections, fission spectrum and averaged number of neutron per fission) have been perturbed, e.g. all isotopes available in both the respective covariance libraries and the CASMO-5 library. The evolution of the uncertainty estimates with exposure is complemented with sensitivity analysis to determine the main contributors to the uncertainty. The Pearson coefficient defined between the model output and a given input is used in this work to assess the part of the variance in the model output coming from the considered input uncertainty. It is a very promising measure of sensitivity as it is computationally cheap even though it assumes linearity of the output with respect to input perturbations. The evolution of the uncertainty with exposure, both in terms of trends and magnitude are however very different. Sensitivity analysis allows determining why the trends and magnitudes are different.
\end{abstract}

KEYWORDS: covariance matrices, depletion uncertainty quantification, CASMO-5, sensitivity analysis

${ }^{*}$ corresponding author 


\section{INTRODUCTION}

At the Laboratory of Reactor physics and Thermal-Hydraulics (LRT) of the Paul Scherrer Institut (PSI), Uncertainty Quantification (UQ) methods have been implemented for some years around the CASMO-5 code using a set of Perl-based scripts named Shark-X. Such UQ is required for providing confidence bounds to the Calculation to Experiment (C/E) comparisons when comparing code outcomes with Post Irradiation Examination experimental data. The source of input uncertainty has a non-negligible impact in this process, as it affects the interpretation of the discrepancies between simulation and measurements through the magnitude of the computational uncertainty.

While the expectation values of the nuclear data are predictive (nuclear data evaluations have been tested against benchmarks for decades), the same cannot be said for their evaluated covariances. They suffer from a range of issues: the mean values are very often adjusted on integral experiments but this is not reflected in the covariance; the uncertainty is usually increased to account for experimental and modeling biases; and cross-correlations between experiments are most of the time neglected; as a result the evaluated covariances will lead to large computational uncertainties compared to their experimental counterparts.

More than a physically accurate estimation of the computational uncertainty, this work aims at assessing the range of possible computational uncertainties that can be obtained with various source of covariance information while using for the same computational model (CASMO-5) and for quantities of interest relevant to burnup credit applications ( $k_{i n f}$, nuclide concentrations). Due to space restrictions, only the $k_{\text {inf }}$ results are covered in the manuscript; results for the nuclide concentrations will be presented at the meeting. The UQ results are complemented with sensitivity analysis, which provides information on the major contributors to the computational uncertainty. This information is less sensitive to the drawbacks of the covariance matrices listed above.

Finally, even though depletion calculations are envisioned, fission yields uncertainties are not considered as no covariance for them is available even in the latest evaluations. As a result, only the actinides concentration uncertainty is analyzed here as a large part of the fission products uncertainty is not accounted for.

\section{PROBLEM DESCRIPTION}

In this section, the various source of covariance information considered are described. The methods to perform the uncertainty propagation and sensitivity analysis are summarized. The last subsection provides a description of the assembly model on which the effect of the various sources of covariances is assessed.

\subsection{Nuclear Data Input Uncertainty}

In this work, the nuclear data input uncertainties are composed of the cross-section channels used in CASMO-5 (elastic and inelastic scattering, n,2n, capture and fission), neutron multiplicity $(\bar{\nu})$ and fission spectrum $(\chi)$.

Concerning the neutron multiplicity the total nubar $(\bar{\nu})$ VCM (MT452) are considered and not the prompt nubar (MT456). The covariance matrices of the recent ENDFB-VIII.0 [1], JEFF-3.3 [2] 
evaluations are considered as well as the more widely distributed ones, JENDL-4.0 [3], ENDF/BVII.1 [4], Scale 6.1 [5] and Scale 6.2 [6]. The covariance matrices are produced in 19 energy-group format using the module ERRORR of NJOY [7] for the JEFF, ENDF/B and JENDL evaluated files. The coverx files available in the respective distributions of Scale 6.1 and 6.2 are used directly. Originally provided in a 44 energy-group (respectively 56) structure, the SCALE covariances are collapsed to the 19-energy group structure of CASMO-5 using the Angelo Lambda code [8].

The number of uncertain nuclide-reaction pairs included in each covariance matrices is listed in Table 1.

Table 1: Content of the covariance matrices. The number of available nuclides represents the number of ENDF files with a MF 33 record. The perturbed nuclides are the ones for which at least one reaction channel has been perturbed in the CASMO-5 library.

\begin{tabular}{|c|c|c|c|}
\hline $\begin{array}{c}\text { Covariance } \\
\text { Matrix }\end{array}$ & $\begin{array}{c}\text { Number of } \\
\text { available nuclides }\end{array}$ & $\begin{array}{c}\text { Number of } \\
\text { perturbed nuclides }\end{array}$ & $\begin{array}{c}\text { Number of nuclides } \\
\text { reaction pairs }\end{array}$ \\
\hline ENDF/B-VIII.0 & 220 & 132 & 349 \\
\hline ENDF/B-VII.1 & 183 & 135 & 355 \\
\hline JEFF-3.3 & 442 & 245 & 532 \\
\hline JENDL 4.0 & 99 & 63 & 214 \\
\hline Scale 6.1 & 385 & 328 & 679 \\
\hline Scale 6.2 & 375 & 328 & 732 \\
\hline
\end{tabular}

\subsection{Uncertainty Quantification}

The uncertainty propagation calculations have been carried out with the SHARK-X [9-11] platform. It provides two major approaches for propagating nuclear data uncertainties. The first ones relies on the calculation of sensitivity coefficients through direct perturbation. Those coefficients are then folded with the covariance matrices through the use of the sandwich rule to compute the uncertainty. The second method relies on Stochastic Sampling (SS).

The perturbations to the cross-sections are introduced in the CASMO-5 code through an in-house routine added to the code. Even though capabilities to do so have been implemented in Shark-X recently [12], the implicit effect (corresponding to the perturbation of the self-shielding factor) is not taken into account in this study.

Because of the large number of uncertain inputs involved in the depletion calculations, the direct perturbation approach is not convenient due to its high computational cost. Consequently, the SS method is used. The SS method requires the perturbation of input data considered as random variables with presumed probability distribution functions. SS calculations consist in the process of assigning distributions to inputs, sampling the inputs (accounting for possible correlations between the inputs), performing independent calculations with each sample, and statistically analyzing the 
distribution of outputs. For the sampling of correlated inputs, a Cholesky-like decomposition is applied to the covariance matrix with a random normal multivariate sampler. For each source of covariance considered, 1'000 CASMO-5 output calculations are performed.

\subsection{Sensitivity Analysis}

For the purpose of identifying the inputs responsible for the computational uncertainty, various methods for sensitivity analysis (SA) have been implemented in Shark-X [13,14]. They are making use of the calculations already performed, e.g. no additional run is needed. Even though uncertainty quantification through the use of random sampling, is relatively straightforward, SA based on those random samples is difficult due to the potential non-linearity of the model, to the large number of inputs, as well as to their correlations. Given the low number of samples available (their computational cost is large), the method based on the determination of Pearson correlation coefficients is used in the present work. Such approach has been used extensively in the past by the so-called "GRS method" [15]. Both first order and total sensitivity indices can be determined. No assumption is made with respect to the probability distribution functions of the input/output. However linearity of the output with respect to input perturbations is assumed.

In statistics, the Pearson correlation $\left(r_{p}\right)$ between two random variables $X_{i}$ and $Y$, is computed as shown in Eq 1.

$$
r_{p}=\frac{\sum_{j=1}^{N}\left(x_{i}^{j}-\bar{X}_{i}\right)\left(y^{j}-\bar{Y}\right)}{\sqrt{\sum_{j=1}^{N}\left(x_{i}^{j}-\bar{X}_{i}\right)^{2}} \sqrt{\sum_{j=1}^{N}\left(y^{j}-\bar{Y}\right)^{2}}}
$$

where $i$ is the input index and $j$ the sample index out of a total of $N$ samples. $\bar{X}_{i}$ and $\bar{Y}$ are the means of the random variables $X_{i}$ and $Y$.

$r_{p}^{2}$ can be interpreted as the coefficient of determination of the relationship between $\mathrm{Y}$ and $X_{i}$ assuming a linear model between $X_{i}$ and Y [16]. It represents the fraction of the variance of $\mathrm{Y}$ which is explained from approximating $\mathrm{Y}$ by a linear combination of the $X_{i}$. This interpretation can be used to estimate the first order sensitivity index of the input parameter $X_{i}$ :

$$
S_{X_{i}}=r_{p}^{2}
$$

Those first order sensitivity indices can be generalized to multiple correlation coefficients which correspond to groups of input parameters [15].Assuming a group of parameters $X_{(1)}=\left(X_{1}, \ldots, X_{k}\right)$ , the multiple correlation coefficient is defined as 2.

$$
R_{(1)}^{2}=\left(r_{p}\left(Y, X_{1}\right), \ldots, r_{p}\left(Y, X_{k}\right)\right) \Sigma_{X_{(1)}}^{-1}\left(r_{p}\left(Y, X_{1}\right), \ldots, r_{p}\left(Y, X_{k}\right)\right)^{T}
$$

where $\operatorname{rp}\left(Y, X_{i}\right)$ is the correlation coefficient between $\mathrm{Y}$ and $X_{i}, \Sigma_{X_{(1)}}^{-1}$ is the inverse of the covariance matrix for the group of parameters $X_{(1)}$.

The first order sensitivity index for a group of parameters can be defined as $S_{X_{(1)}}=R_{(1)}^{2}$ and the total index for a group of parameters as $S_{X_{(1)}}^{T}=R^{2}-R_{(1)}^{2} \cdot R_{(1)}^{2}$ is the multiple correlation coefficient between $\mathrm{Y}$ and the remaining group of input parameters (the ones not included in $X_{(1)}$ ). $R^{2}$ is the total correlation coefficient between $\mathrm{Y}$ and the full set of input parameters $\mathrm{X}$. 


\subsection{Irradiated Fuel Sample}

One sample of the LWR-PROTEUS Phase II program [17] is used to illustrate the effects of considering various sources of nuclear data uncertainty during depletion calculations. It consists of a segment of PWR UO2 fuel rod irradiated in the Gösgen nuclear power plant (Switzerland) for two cycles up to a burnup of $38 \mathrm{MWd} / \mathrm{kgU}$ with a 25 days shutdown between the cycles. A validation of CASMO-5 is currently on-going at PSI[18] including the analysis of these samples and several others, one goal being to assess the transition from the predecessor CASMO-4E code to CASMO-5. The 2-D CASMO-5 (v.2.03.00) assembly model (including depletion/decay histories) of [18] is employed for this UQ analysis, but are performed with a multi-group ENDF/BVII.0 (e7r0.125.586) [19] based library. The irradiation history is modeled through steps functions for various state parameters including boron concentration, fuel and moderator temperatures and power.

\section{RESULTS}

Due to space restrictions, only the $k_{\text {inf }}$ results are discussed here; results for the nuclide concentrations will be presented at the meeting. The UQ results for the various sources of covariance matrices are analyzed first; the sensitivity analysis outcomes are provided next.

\section{1. $\mathbf{k}_{\text {inf }}$ uncertainty quantification}

The evolution of the $\mathrm{k}_{\text {inf }}$ relative uncertainty $(1 \sigma$ in $\mathrm{pcm})$ with respect to the time spent in the reactor is shown in Figure 1. The magnitudes as well as the trends in $\mathrm{k}_{\text {inf }}$ relative uncertainty are widely different depending on the considered source of nuclear data covariance.

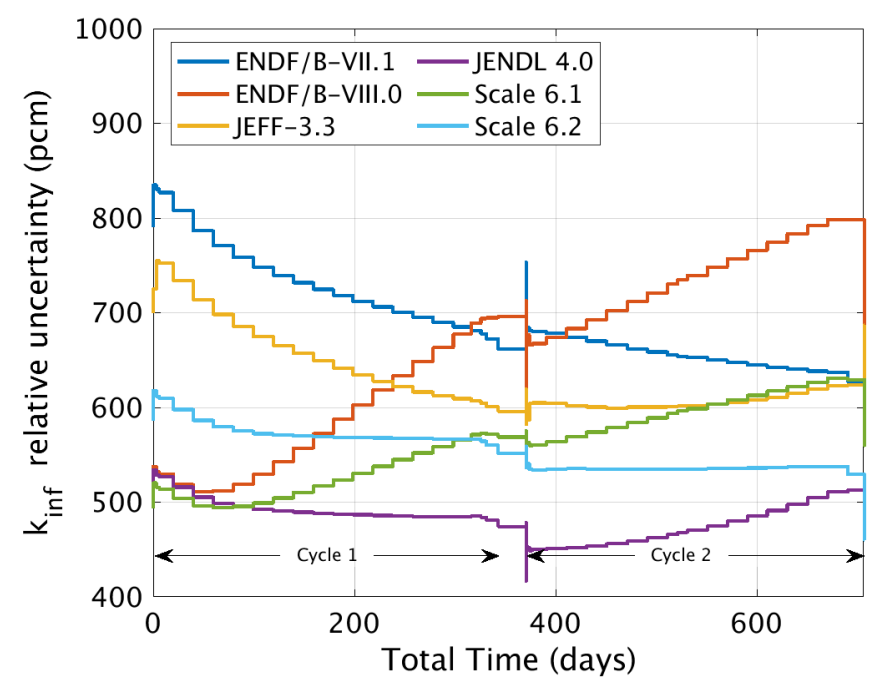

Figure 1: Evolution of the $\mathbf{k}_{i n f}$ relative standard deviation with exposure. Various covariance matrices are considered

JENDL 4.0 produces the lowest uncertainty estimate but this is due to reduced input uncertainties and not to the much lower number of perturbed nuclide-reaction pairs (see Table 1). The Scale 
covariance matrices tend to also produce lower uncertainty estimates albeit with different trends: Scale 6.2 produces larger uncertainties than Scale 6.1 initially, but its evolution with exposure is fairly flat throughout the two cycles while the Scale $6.1 \mathrm{k}_{\text {inf }}$ uncertainty is similar to the JENDL 4.0 one for fresh fuel but increases steadily throughout fuel irradiation.

The different behaviors of Scale 6.1 and 6.2 are well known and mostly related to the change in $\bar{\nu}$ uncertainties for ${ }^{239} \mathrm{Pu}$ and ${ }^{235} \mathrm{U}$ in Scale 6.2 [20]. It should be noted that the results of Scale 6.0 reported in [21] are very similar to ones of the Scale 6.1 curve.

The ENDF/B-VIII.0 trend is similar to ones of Scale 6.1 with an even steeper increase as a function of exposure.

Finally ENDF/B-VII.1 and JEFF-3.3 produce initially larger $\mathrm{k}_{\text {inf }}$ uncertainties. But their trends are decreasing with exposure during the first cycle. Then for ENDF/B-VII.1, the $\mathrm{k}_{\text {inf }}$ uncertainty keeps decreasing while it increases for JEFF-3.3 towards the end of the second irradiation cycle. The surprising decreasing trend of JEFF-3.3 is due to not having average nubar covariances for ${ }^{239} \mathrm{Pu},{ }^{235} \mathrm{U}$ and ${ }^{238} \mathrm{U}$ in JEFF-3.3: only the prompt nubar covariances are available. It should be noted that in this work, and for ${ }^{235} U$ only, it was assumed that prompt and averaged nubar covariances are the same leading to large uncertainties for $\mathrm{k}_{\text {inf }}$ at low exposures. However, the potentially large source of uncertainty due to ${ }^{239} \mathrm{Pu}$ at larger exposure is not considered.

Next sensitivity analysis is performed in order to understand better the trends observed in the outcomes of the UQ calculations.

\section{2. $\mathbf{k}_{\text {inf }}$ sensitivity analysis}

No additional calculations was performed; the outcomes of the study presented below required solely post processing of the existing output samples. The energy groups of a given nuclidereaction pair are grouped together; the sensitivity of the corresponding nuclide-reaction pair is estimated using the multiple correlation coefficient defined in Eq. 3. The number of samples available is much larger than the number of input parameter grouped together for the first order sensitivity index (19), but not enough for the total sensitivity index (K-19) where K is the total number of uncertain input which is much larger than 1'000 as reported in Table 1. Consequently, the determination of the total sensitivity coefficients are not considered further.

The evolution of the first order sensitivity indices for $k_{\text {inf }}$ during irradiation are shown in Figure 2. For the sake of conciseness, only the most recent sources of covariances are considered (ENDF/BVIII.0, JEFF-3.3, Scale 6.2 and JENDL 4.0). Moreover, only the sensitivity indices for the 3 nuclide-reaction pairs contributing the most to the integral parameter uncertainty at a given time during the irradiation are shown.

For both JENDL 4.0 and Scale 6.2, the main contributors to the uncertainty are ${ }^{238} \mathrm{U}$ capture followed by ${ }^{235} \mathrm{U} \bar{\nu}$ initially. Then during the second irradiation cycle, the increase in $\mathrm{k}_{\text {inf }}$ uncertainty in JENDL 4.0 is due to the build-up of ${ }^{241} \mathrm{Pu}$ and its large capture cross section uncertainty (see Figure $3 \mathrm{a}$ and $3 \mathrm{~b}$ ). Using the Scale 6.2 covariances, the uncertainty estimates remain constant as the decreasing importance of ${ }^{238} U$ capture and ${ }^{235} \mathrm{U} \bar{\nu}$ is compensated by the increasing importance of the fission and capture cross sections of ${ }^{239} \mathrm{Pu}$ (see Figure $2 \mathrm{~d}$ ). The decreasing importance of ${ }^{235} \mathrm{U}$ can be understood by its consumption while the decreasing importance of ${ }^{238} \mathrm{U}$ capture is a well known phenomenon related to the build up of ${ }^{239} \mathrm{Pu}$.

In JEFF-3.3, the capture of ${ }^{238} \mathrm{U}$ does not play a significant role in the $\mathrm{k}_{\text {inf }}$ uncertainty due to its low input uncertainty. The decreasing trend in $\mathrm{k}_{\text {inf }}$ uncertainty is due to the consumption of ${ }^{235} \mathrm{U}$. 


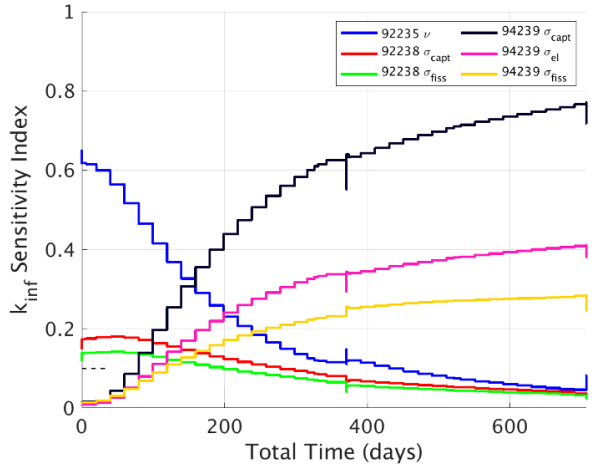

(a) ENDF/B-VIII.0

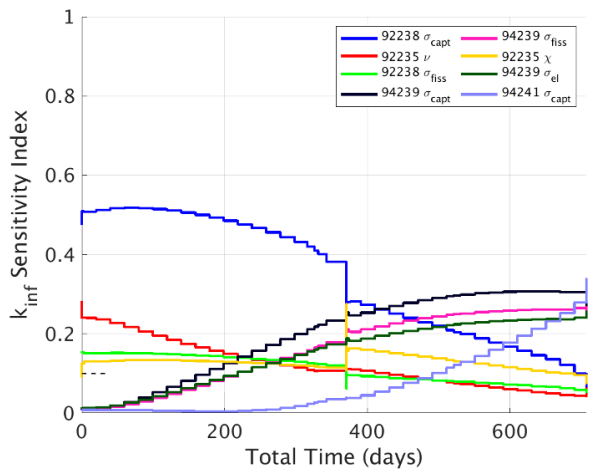

(c) JENDL 4.0

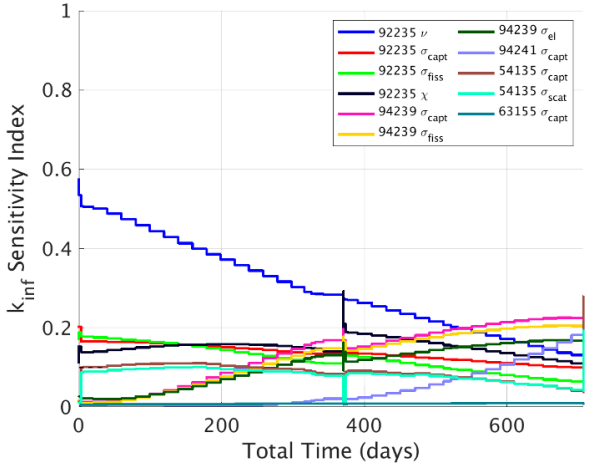

(b) JEFF-3.3

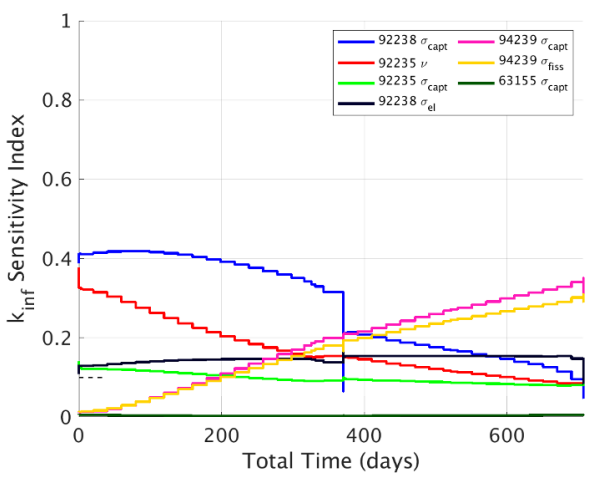

(d) Scale 6.2

Figure 2: Evolution of the sensitivity indices for the 3 nuclides reaction pairs contributing the most to $k_{i n f}$ uncertainty for various sources of covariance.

The increase towards the end of the second cycle is due to the accrued importance of the capture and fission cross sections of ${ }^{239} \mathrm{Pu}$ as well as the capture cross sections of ${ }^{241} \mathrm{Pu}$.

Finally for ENDF/B-VIII.0, the increase of $\mathrm{k}_{\text {inf }}$ uncertainty with exposure is due to the buildup of ${ }^{239} \mathrm{Pu}$ and the large uncertainties of capture, fission and elastic scattering cross sections. Such outcomes are confirmed by looking at the evolution of the variance with energy of the cross sections. Due to reduced space, only the case ${ }^{241} \mathrm{Pu}$ was presented in the paper (see Figure $3 b$ ). The others will be shown during the presentation.

\section{CONCLUSIONS}

This paper describes the effect of input uncertainties on k-inf. The nuclear data under consideration are the cross-sections, fission spectrum and neutron multiplicities available in novel (ENDFBVIII.0, JEFF-3.3) as well as more widely distributed (JENDL-4.0, ENDF/B-VII.1, Scale 6.1 and Scale 6.2) covariance matrices. The Pearson coefficient defined between the model output and a given input is used in this work to assess the part of the variance in the model output coming from the considered input uncertainty.

The first difference between the various sources of covariance is the large differences in terms of coverage of the relevant nuclide reaction pairs for which input uncertainties are available. The 


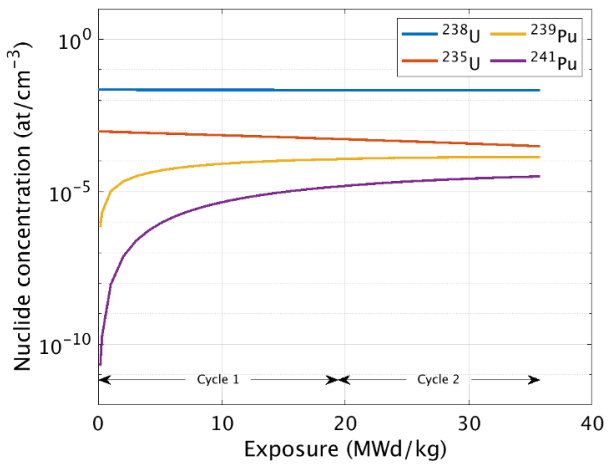

(a) Evolution of the nuclide densities for important actinides

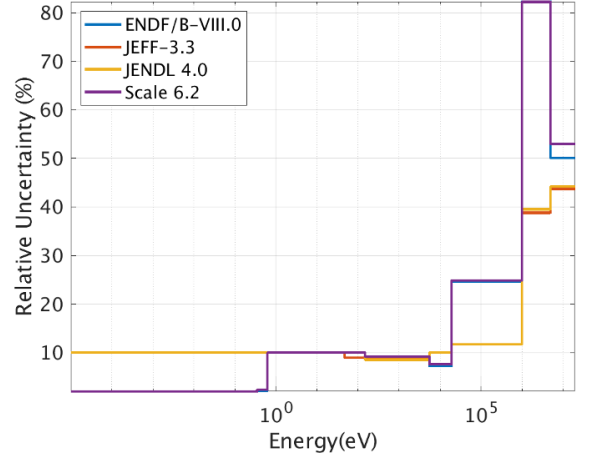

(b) standard deviation of ${ }^{241} \mathbf{P u} \sigma_{\text {capture }}$

Figure 3: Additional information about the JENDL 4.0 results.

novel nuclear data libaries have made progress towards providing systematically this information. However, gaps still exist.

More concerning are the wide variety of magnitude, trends and major contributors to the uncertainties of standard integral parameters. Those differences are observed even with the most recent source of nuclear data uncertainties. They are especially obvious during burnup calculations as a wider range of nuclide-reaction pairs are involved.

Even though a large international effort (CIELO [22]) has been carried out to provide consistent covariance information for a reduced number of isotopes, including ${ }^{238} \mathrm{U}$, this work demonstrates that large discrepancies for resulting integral parameters uncertainty estimates are still observed, involving those isotopes. It seems that the road to predictive uncertainty estimation for integral parameters is still long.

\section{REFERENCES}

[1] D. A. Brown, M. B. Chadwick, R. Capote, A. C. Kahler, A. Trkov, M. W. Herman, A. A. Sonzogni, Y. Danon, A. D. Carlson, M. Dunn, D. L. Smith, G. M. Hale, G. Arbanas, R. Arcilla, C. R. Bates, B. Beck, B. Becker, F. Brown, R. J. Casperson, J. Conlin, D. E. Cullen, M. A. Descalle, R. Firestone, T. Gaines, K. H. Guber, A. I. Hawari, J. Holmes, T. D. Johnson, T. Kawano, B. C. Kiedrowski, A. J. Koning, S. Kopecky, L. Leal, J. P. Lestone, C. Lubitz, J. I. Márquez Damián, C. M. Mattoon, E. A. McCutchan, S. Mughabghab, P. Navratil, D. Neudecker, G. P. Nobre, G. Noguere, M. Paris, M. T. Pigni, A. J. Plompen, B. Pritychenko, V. G. Pronyaev, D. Roubtsov, D. Rochman, P. Romano, P. Schillebeeckx, S. Simakov, M. Sin, I. Sirakov, B. Sleaford, V. Sobes, E. S. Soukhovitskii, I. Stetcu, P. Talou, I. Thompson, S. van der Marck, L. Welser-Sherrill, D. Wiarda, M. White, J. L. Wormald, R. Q. Wright, M. Zerkle, G. Žerovnik, and Y. Zhu. "ENDF/B-VIII.0: The 8th Major Release of the Nuclear Reaction Data Library with CIELO-project Cross Sections, New Standards and Thermal Scattering Data." Nuclear Data Sheets, volume 148, pp. 1-142 (2018).

[2] “JEFF-3.3." (2018). URL https://www.oecd-nea.org/dbdata/jeff/jeff33/index.html.

[3] “JENDL4.0 Update 1 Evaluated Nuclear Data File.” URL http://wwwndc.jaea.go.jp/jendl/ j40/update/. 
[4] M. Chadwick, M. Herman, P. Obložinský, M. Dunn, Y. Danon, a.C. Kahler, D. Smith, B. Pritychenko, G. Arbanas, R. Arcilla, R. Brewer, D. Brown, R. Capote, a.D. Carlson, Y. Cho, H. Derrien, K. Guber, G. Hale, S. Hoblit, S. Holloway, T. Johnson, T. Kawano, B. Kiedrowski, H. Kim, S. Kunieda, N. Larson, L. Leal, J. Lestone, R. Little, E. McCutchan, R. MacFarlane, M. MacInnes, C. Mattoon, R. McKnight, S. Mughabghab, G. Nobre, G. Palmiotti, a. Palumbo, M. Pigni, V. Pronyaev, R. Sayer, a.a. Sonzogni, N. Summers, P. Talou, I. Thompson, a. Trkov, R. Vogt, S. van der Marck, a. Wallner, M. White, D. Wiarda, and P. Young. "ENDF/B-VII.1 Nuclear Data for Science and Technology: Cross Sections, Covariances, Fission Product Yields and Decay Data." Nuclear Data Sheets, volume 112(12), pp. 2887-2996 (2011). URL http://linkinghub.elsevier.com/retrieve/pii/S009037521100113X.

[5] ORNL. "SCALE: A Comprehensive Modeling and Simulation Suite for Nuclear Safety Analysis and Design." (2011).

[6] B. Rearden. "Overview of SCALE 6.2." In Proceedings of ANS NCSD, Wilmington, NC. Wilmington, North Carolina, USA (2013).

[7] R. E. Macfarlane, D. W. Muir, R. M. Boicourt, and A. C. Kahler. "The NJOY Nuclear Data Processing System, Version 2012.” LANL Report, (LA-UR-12-27079) (2012).

[8] I. A. Kodeli. "VITAMIN-J/COVA/EFF-3 cross-section covariance matrix library and its use to analyse benchmark experiments in sinbad database." Fusion Engineering and Design, volume 75-79(SUPPL.), pp. 1021-1025 (2005).

[9] O. Leray, H. Ferroukhi, M. Hursin, A. Vasiliev, and D. Rochman. "Methodology for core analyses with nuclear data uncertainty quantification and application to Swiss PWR operated cycles." Annals of Nuclear Energy, volume 110 (2017).

[10] W. Wieselquist, T. Zhu, A. Vasiliev, and H. Ferroukhi. "PSI Methodologies for Nuclear Data Uncertainty Propagation with CASMO-5M and MCNPX: Results for OECD / NEA UAM Benchmark Phase I." Science and Technology of Nuclear Installations, p. 15 (2013).

[11] M. Hursin, M. Scriven, G. Perret, and A. Pautz. "Uncertainty quantification and representativity analysis of LWR-PROTEUS Phase III experiments using SHARKX." Annals of $\mathrm{Nu}$ clear Energy, volume 91, pp. 48-58 (2016). URL http://www.scopus.com/inward/record. url?eid=2-s2.0-84954444565\{\\&\}partnerID=tZOtx3y1.

[12] M. Hursin, S. Pelloni, A. Vasiliev, and H. Ferroukhi. "Treatment of the implicit effect in Shark-X." Annals of Nuclear Energy, volume 138 (2020).

[13] M. Hursin, D. Siefman, G. Perret, D. Rochman, A. Vasiliev, and H. Ferroukhi. "Determination of Sobol Sensitivity Indices for Correlated Inputs with SHARK-X." In Proceedings of PHYSOR 2018. Cancun, Mexico (2018).

[14] M. Hursin, D. J. Siefman, G. Perret, and A. Pautz. "Representativity analysis of the LWRPROTEUS Phase II experiments using SHARK-X stochastic sampling method." In American Nuclear Society, editor, Best Estimate Plus Uncertainty International Conference (BEPU 2018). American Nuclear Society, Lucca, Italy (2018).

[15] H. Glaeser. "GRS method for uncertainty and sensitivity evaluation of code results and applications." Science and Technology of Nuclear Installations, volume 2008 (2008).

[16] T. Most. "Variance-based sensitivity analysis in the presence of correlated input variables." In 5th Int. Conf. Reliable Engineering Computing (REC), , pp. 13-15. Brno, Czec (2012).

[17] P. Grimm. "Burnup Calculations and Chemical Analysis of Irradiated Fuel Samples Studied in LWR-PROTEUS Phase II.” In Physor 2006 American Nuclear Society's Topical Meeting on Reactor Physics, pp. 1-10 (2006). 
[18] P. Grimm, M. Hursin, G. Perret, D. Siefman, and H. Ferroukhi. "CASMO-5 ANALYSIS OF REACTIVITY WORTHS OF BURNT PWR FUEL SAMPLES MEASURED IN LWRPROTEUS PHASE II." In PHYSOR 2016 - Unifying Theory and Experiments in the 21st Century, pp. 1-5. Sun Valley Resort, Sun Valley, Idaho, USA (2016).

[19] M. Chadwick, P. Obložinský, M. Herman, N. Greene, R. McKnight, D. Smith, P. Young, R. MacFarlane, G. Hale, S. Frankle, a.C. Kahler, T. Kawano, R. Little, D. Madland, P. Moller, R. Mosteller, P. Page, P. Talou, H. Trellue, M. White, W. Wilson, R. Arcilla, C. Dunford, S. Mughabghab, B. Pritychenko, D. Rochman, a.a. Sonzogni, C. Lubitz, T. Trumbull, J. Weinman, D. Brown, D. Cullen, D. Heinrichs, D. McNabb, H. Derrien, M. Dunn, N. Larson, L. Leal, a.D. Carlson, R. Block, J. Briggs, E. Cheng, H. Huria, M. Zerkle, K. Kozier, a. Courcelle, V. Pronyaev, and S. van der Marck. "ENDF/B-VII.0: Next Generation Evaluated Nuclear Data Library for Nuclear Science and Technology." Nuclear Data Sheets, volume 107(12), pp. 2931-3060 (2006). URL http://linkinghub.elsevier.com/retrieve/pii/ S0090375206000871.

[20] W. J. Marshall, M. L. Williams, D. Wiarda, B. T. Rearden, M. E. Dunn, D. E. Mueller, J. B. Clarity, and E. L. Jones. "Development and testing of Neutron cross-section covariance data for SCALE 6.2." In ICNC 2015 - International Conference on Nuclear Criticality Safety, pp. 1213-1225. Charlotte, North Carolina, USA (2015).

[21] O. Leray, D. Rochman, P. Grimm, H. Ferroukhi, A. Vasiliev, M. Hursin, G. Perret, and A. Pautz. "Nuclear data uncertainty propagation on spent fuel nuclide compositions." Annals of Nuclear Energy, volume 94, pp. 603-611 (2016).

[22] OECD/NEA. "CIELO Pilot Project." 